\title{
Broadening the problem agenda of biological individuality: individual differences, uniqueness and temporality
}

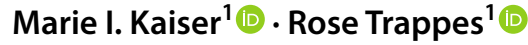

Received: 5 October 2019 / Accepted: 16 February 2021 / Published online: 3 March 2021

(c) The Author(s) 2021

\begin{abstract}
Biological individuality is a notoriously thorny topic for biologists and philosophers of biology. In this paper we argue that biological individuality presents multiple, interconnected questions for biologists and philosophers that together form a problem agenda. Using a case study of an interdisciplinary research group in ecology, behavioral and evolutionary biology, we claim that a debate on biological individuality that seeks to account for diverse practices in the biological sciences should be broadened to include and give prominence to questions about uniqueness and temporality. We show that broadening the problem agenda of biological individuality draws attention to underrecognized philosophical issues and discussions and thereby organizes and enriches the existing debate.
\end{abstract}

Keywords Biological individuality · Problem agenda - Uniqueness · Temporality · Individual differences $\cdot$ Identity $\cdot$ Temporal stability

\section{Introduction}

The philosophical debate on biological individuality often focuses on questions of how to identify biological individuals (e.g., Clarke 2010; Kovaka 2015; Lidgard and Nyhart 2017; Wilson and Barker 2018). What counts as a biological individual? How can biological individuals be defined, and which criteria can be used to pick out biological individuals? The sheer difficulty of identifying individuals in biological reality has generated the debate on biological individuality centered on questions about individuation and related questions of demarcation, parthood, and unity. We follow Scott Lidgard and Lynn Nyhart in characterizing this discussion in terms of a "problem agenda" $(2017,45)$, consisting of multiple interconnected questions provoked by closely related phenomena. Adopting this terminology, we argue that the problem agenda of biological individuality is broader than often thought.

Marie I. Kaiser

kaiser.m@uni-bielefeld.de

1 Department of Philosophy, Bielefeld University, Bielefeld, Germany 
Our aim to broaden the problem agenda of biological individuality stems from our work within the DFG-funded Collaborative Research Centre "A Novel Synthesis of Individualisation across Behaviour, Ecology and Evolution: Niche Choice, Niche Conformance, Niche Construction" (in the following 'CRC'). The CRC is a large interdisciplinary research group involving ecologists, behavioral biologists, evolutionary biologists, and theoretical biologists, in which we participate as resident philosophers. The biologists in the CRC address the task of identifying, studying, and explaining individual differences, such as differences in personalities of individual animals (e.g., boldness, optimism/pessimism), and individualized niches (individuals having different ecological niches). The role of the philosophers in this research group is to collaborate with the biologists to refine and clarify central concepts and working hypotheses as well as to develop a sound and empirically fruitful theoretical framework for the group's research.

The biologists in our case study do not ask how to identify biological individuals, nor how to determine what their parts are, where their boundaries lie, or what holds together an individual's parts. Questions about identification, demarcation, parthood, and unity may be urgent in other research contexts, including within behavioral ecology (Smith-Ferguson and Beekman 2019). Hence, we do not believe that the recent philosophical debate on biological individuality is irrelevant for practicing biologists (Kovaka 2015). Nevertheless, the biologists in our case study focus on different phenomena and raise different questions about biological individuality than those which are most prominent in the philosophical debate. Accordingly, encompassing a full range of biological practices requires paying attention to these other questions.

Based on our case study, we identify two sets of questions about biological individuality that are often underrecognized in the philosophy of biology. First, the CRC's questions about how individuals differ from one another raise philosophical questions about what makes biological individuals unique. ${ }^{1}$ Second, the CRC's questions about changes and stability invite questions about how biological individuality changes over different timescales as well as how much and what sorts of temporal stability is required. Although questions about uniqueness and temporality have been asked by philosophers of biology, they have received little systematic recognition and treatment. In addition, some uniqueness and temporality questions have been neglected altogether. We therefore argue that questions about uniqueness and temporality should be recognized as central parts of the problem agenda of biological individuality, especially because including them will bring the philosophical debate closer to a broader range of biological practices.

In "The problem agenda of biological individuality" Section we introduce the problem agenda of biological individuality. We present our case study in "New trends in studying biological individuality" Section, highlighting how the researchers investigate individual differences. In "Uniqueness questions" and "Temporality

\footnotetext{
1 In this paper, we follow the biologists in the CRC and use the term 'individual' to refer only to organisms. However, this is not to say that non-organismal biological individuals do not raise interesting questions about uniqueness and temporality.
} 
questions" Sections we explicate the philosophical questions provoked by the CRC's work, including questions about uniqueness and about temporality, and argue that these questions belong to the problem agenda of biological individuality. We conclude in "Conclusions" Section with a reconstruction of the extended problem agenda of biological individuality.

\section{The problem agenda of biological individuality}

This section introduces the main questions addressed in the recent philosophical debate on biological individuality. Our aim is not to reconstruct the course of the debate and its major positions and arguments. Rather, we shed light on the central questions of the debate and how they are linked together in the problem agenda of biological individuality ("Identifying biological individuals" and "Questions of identification, demarcation, parthood, and unity" Sections). We also explain why biological individuality poses problems to biologists and philosophers ("Origins of the problematic nature of biological individuality" Section). We focus on the most dominant questions in the debate on biological individuality. Other questions have arisen in relation to biological individuality, but they are typically peripheral and are not treated in a systematic way.

\section{Identifying biological individuals}

'What is a biological individual?', or 'What makes something a biological individual?', is a question that takes center stage in the recent philosophical debate about biological individuality. This question asks for a characterization or definition of biological individuality. Answers are often given in terms of certain features or properties that biological individuals must have, such as spatial continuity, functional autonomy, or genetic homogeneity. It is also common to refer to processes that individuals must undergo, such as reproduction, natural selection, and cooperation or conflict. These properties and processes give rise to a plurality of identification criteria (see e.g., Clarke 2010, 315-320; Boulter 2013, 81; Lidgard and Nyhart $2017,19-21)$. In turn, these criteria-alone or in combination-constitute the core of different concepts of biological individuality and can be used to pick out different kinds of biological individuals, such as evolutionary, developmental, immunological, and ecological individuals.

Criteria and concepts of biological individuality allow us to identify which entities in the world are "genuine individuals" (Huneman 2014, 362). In particular, they help to distinguish biological individuals from mere assemblies or groups of biological individuals on the one hand, and from parts of biological individuals on the other (Pepper and Herron 2008, 622; Bouchard and Huneman 2013, 6; Wilson and 
The Problem Agenda of Biological Individuality

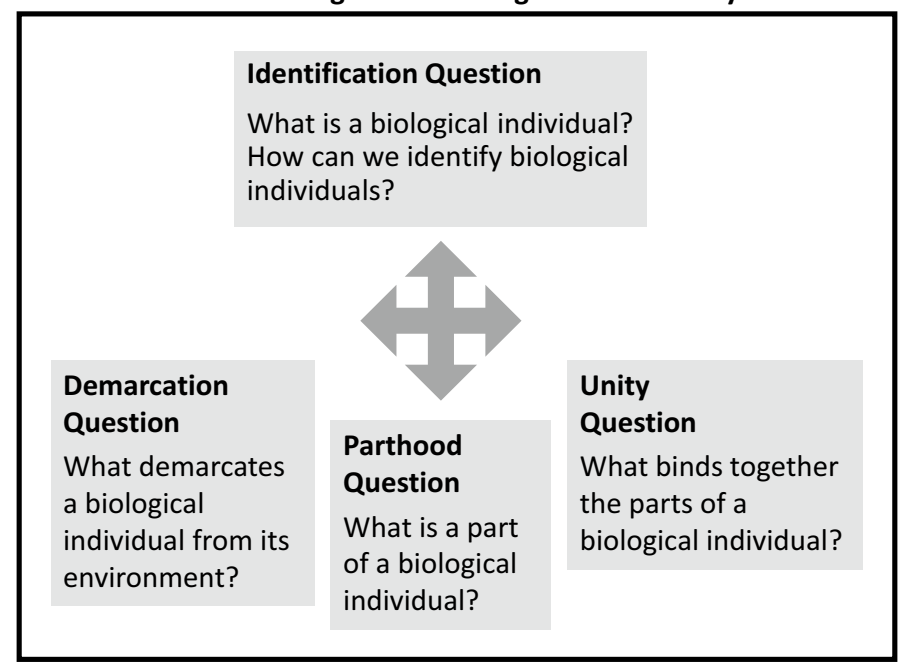

Fig. 1 The Existing Problem Agenda of Biological Individuality. Central questions in the problem agenda are linked by various interrelations, indicated by the multi-headed arrow. Less prominent questions or less systematic philosophical discussions about biological individuality are not depicted

Barker 2018, Sec. 3). ${ }^{2}$ Questions about identifying entities as biological individuals are often called questions of individuation. However, individuation covers more than only picking out individuals in the world. We thus use the term identification to refer specifically to questions concerning the criteria for and activity of picking out biological individuals. The identification question is central to the problem agenda of biological individuality.

Recently, it has been argued that philosophers should not ask "What is a biological individual?", but rather "How do biologists individuate biological individuals, and for what purposes?" (Waters 2018; see also Love and Brigandt 2017; Bueno et al. 2018). We agree that philosophers should pay attention to concrete practices of identifying biological individuals and to the epistemic goals different practices serve. Such practice-based approaches are widespread in the debate on biological individuality. However, even practice-based approaches to biological individuality have centered on identification questions (as well as questions of demarcation, parthood, and unity, see "Questions of identification, demarcation, parthood, and unity" Section).

\footnotetext{
${ }^{2}$ This claim is compatible with the widely acknowledged fact that biological individuality occurs on multiple levels of organization (Gould and Lloyd 1999, 11,906; Guay and Pradeu 2015, 7; Lidgard and Nyhart 2017, 32).
} 


\section{Questions of identification, demarcation, parthood, and unity}

Identifying biological individuals is closely linked to other tasks, such as demarcating a biological individual from its environment (including from other individuals), decomposing the biological individual into parts, and specifying the kind of unity that holds an individual's parts together. This is why discussions about biological individuality are often interwoven with discussions about part-whole relations, demarcation, decomposition, constitution, levels, integration, and unity. Figure 1 depicts the identification question distinguished from three other central questions: questions about demarcation, parthood, and unity (see Kaiser 2018).

Despite these four questions being distinct, answers to one often imply or presume answers to others. For example, if you think that biological individuals are units of selection, this will have implications for your views on what binds together an individual's parts (e.g., their common fate in selection processes), and when something is a part of a biological individual (e.g., if it engages in a process that contributes to the individual's fitness; Haber 2015; Kaiser 2018). In contrast, if you take functional autonomy as an important criterion of biological individuality, this will predispose you to regard functional integration as crucial for unity and the existence or absence of functional relations as central to determining parts and boundaries. This is not even to mention the complex interrelations among questions of demarcation, parthood, and unity themselves.

Given the interconnectedness of different questions, the debate on biological individuality can be characterized as a problem agenda. A problem agenda, as developed by Alan Love (2005, Ch. 2; 2008; 2016), is a set of interconnected questions generated by a set of related phenomena. While specific phenomena and questions can come in and out of focus in the course of research, Love suggests that the problem agenda furnishes a field with a continuous research program. We believe Lidgard and Nyhart (2017) are correct in suggesting that biological individuality, too, constitutes a problem agenda. The notion of a problem agenda nicely captures the fact that questions about identification, demarcation, parthood, and unity are deeply interconnected.

\section{Origins of the problematic nature of biological individuality}

Why does biological individuality give rise to problems? Recent literature highlights three reasons for the problematic nature of biological individuality. First, there is a clash between our everyday intuitions and biological reality (e.g., Wilson 1999; Bouchard and Huneman 2013; Chauvier 2017). On the one hand, our intuitions concerning biological individuals arise from familiar organisms: higher-level animals and especially adult mammals. On the other hand, individuality concepts are usually tasked with accommodating the wide variety of biological individuals studied in the biological sciences. Many of these biological individuals, such as colonial organisms, symbiotic associations, plants, microbes, ecosystems, and clonal species, look "exotic" (Bouchard and Huneman 2013, 3; Chauvier 2017, 1) or "weird" (Huneman 
2014, 362) from the perspective of everyday intuitions. The clash between intuitive individuality concepts and those that capture the diversity of biological individuals is a major source of debate about biological individuality.

Second, in some domains it is very hard to identify biological individuals (Folse and Roughgarden 2010; Clarke 2013; Clarke and Okasha 2013; Huneman 2014). Ellen Clarke refers to such difficult cases as "substantial 'individuation puzzles", (2013, 414). Many of the unintuitive individuals just mentioned are also examples of individuation puzzles. In these "problem cases," it is often not obvious how to delineate discrete biological individuals. This is problematic not only for philosophers but also for practicing biologists, for instance when biologists need to count individuals to make fitness assessments (Clarke 2010, 313).

Third, much recent debate concerns the existence of many different, partly conflicting concepts of biological individuality (J Wilson 1999; Clarke 2010; Clarke and Okasha 2013; Lidgard and Nyhart 2017; Bueno et al. 2018). Extensive lists (e.g., Lidgard and Nyhart 2017, 19-21) impressively illustrate the large number and diversity of proposed identification criteria and concepts of biological individuality, some of which lead to different results when identifying biological individuals (Clarke 2010). The many and contrary biological individuality concepts present problems for both monists and pluralists. Monists aim to find a single consistent, unified and universal concept of biological individuality, which typically prioritizes one kind of identification criterion, such as evolutionary criteria (Godfrey-Smith 2009, 84-86; Clarke 2012). Hence, monists debate which of the multiplicity of concepts of biological individuality is the right one. As Clarke puts it, "there is a choice to be made about which definition [...] to accept" $(2010,315)$. Pluralists, in contrast, endorse the adequacy of many different individuality concepts in different contexts and for different purposes (Love and Brigandt 2017; Lidgard and Nyhart 2017; Bueno et al. 2018; Waters 2018). A common pluralistic view is that there are different kinds of biological individuality-evolutionary, developmental, metabolic, ecological, immunological, and so on (e.g., Pradeu 2016, 766-769). Pluralists face the problem of determining which of the many individuality concepts operate in which contexts, how they relate to each other and which are appropriate for which purposes.

Recognizing that these three reasons all motivate the central questions in the problem agenda, there are nevertheless more questions about biological individuality to be asked. In the next section we introduce our case study, which forms the springboard for our recognition of two underestimated sorts of philosophical questions about biological individuality.

\section{New trends in studying biological individuality}

Our case study highlights an aspect of biological research on individuality that has received little attention in philosophy, the investigation of individual differences. In this section, we explicate the abstract research questions of the biologists in our case study ("The CRC's abstract research questions" Section) and illustrate how these abstract questions are exemplified in specific research questions ("The CRC's specific research questions" Section). We then explain why questions about individual 
differences do not give rise to standard questions about biological individuality ("Individual differences and biological individuality" Section).

\section{The CRC's abstract research questions}

The CRC is a large research center with seventeen projects bringing together ecology, behavioral biology, evolutionary biology, theoretical biology, statistics, and philosophy of biology. What unites these projects is their joint interest in identifying, studying, and explaining individual differences, that is, differences between individual organisms in a population. Especially important are individual differences that are temporally stable and contextually consistent, and that are not attributable to broad categories like age, sex, or morphological type (Dall et al. 2012; Wolf and Weissing 2012; Kaiser and Müller 2021). Different fields study individual differences in their own way, including polymorphism in evolutionary biology (Oliveira et al. 2008), individual specialization in ecology (Bolnick et al. 2003; Araújo et al. 2011; Violle et al. 2012), and animal personality in behavioural biology (Wolf and Weissing 2012; Trillmich et al. 2015; Kaiser and Müller 2021).

Much of the initial scientific work on individual differences has been concerned with shifting the focus from species optima or averages towards recognizing that individual organisms are not identical and interchangeable (Violle et al. 2012; Layman et al. 2015). The CRC moves beyond recording individual differences to ask about ecological, evolutionary, and developmental causes and consequences of individual differences, as well as the general mechanisms that give rise to individual differences. Accordingly, the overall goal of the CRC is "to gain a comprehensive understanding of how individual phenotypes interact with their environment and what the ensuing consequences for ecological and evolutionary processes are."3

The role of the philosophers in the CRC is to clarify central concepts, such as 'individuality,' 'individualized niche,' 'fitness,' 'animal personality,' or 'mechanisms of niche choice, conformance, and construction.' This conceptual philosophical work is strongly informed by biological research and is often undertaken in close collaboration with CRC biologists in joint seminars, workshops, retreats, regular 'concept cloud' meetings, and in joint publications (e.g., Kaiser and Müller 2021). By defining concepts, revealing underlying epistemic and ontological assumptions and scrutinizing working hypotheses, philosophers contribute to developing a sound theoretical framework for the CRC's empirical research. ${ }^{4}$

In our philosophical work we found that the CRC's research can be understood in terms of abstract research questions and the specific research questions that exemplify them. Abstract questions concern the phenomena studied by the CRC on a more general level than any specific project could answer. Based on abstracting

\footnotetext{
3 https://www.uni-bielefeld.de/biologie/crc212/about.html.

${ }^{4}$ Due to our close collaboration with biologists, our philosophical approach is descriptivist rather than revisionist (Smith-Ferguson and Beekman 2019, 1939). Nevertheless, it is worth recognizing that a descriptivist approach does not preclude making normative claims and is compatible with criticizing how biologists use certain terms (Kaiser 2019).
} 
away from specific research organisms, laboratory or field settings, and research interests of individual projects, they express the general goals of the CRC. In contrast, specific questions about individual differences are those asked and addressed by individual projects in the CRC. Since formulating abstract questions based on specific questions involves philosophical interpretation, it is not surprising that the abstract questions in the CRC are closely related to the philosophical questions that we elaborate in "Uniqueness questions" and "Temporality questions" Sections.

We identified three abstract questions concerning individual differences asked in the CRC. First, what are the evolutionary, ecological, and sometimes developmental causes of individual differences? Second, what are the evolutionary and ecological consequences of individual differences? Third, which genetic, transcriptomic, epigenetic, hormonal, and other physiological mechanisms underlie individual differences? Although the biologists may not classify their general research questions in exactly this way, the details of the questions are very similar to how the biologists describe the questions that the CRC addresses.

The first two of these abstract questions are related to the CRC's new framework of so-called $\mathrm{NC}^{3}$ mechanisms (i.e., mechanisms of Niche Construction, Niche Choice, and Niche Conformance). $\mathrm{NC}^{3}$ mechanisms specify the way that individual organisms create, choose, and conform to aspects of the environment (Kaiser and Trappes forthc.). Describing $\mathrm{NC}^{3}$ mechanisms tells us about ecological causes and consequences of individual differences, specifying which ecological entities and activities cause or result from specific individual differences or changes in individual differences. For instance, in Project A04 (Caspers) ${ }^{5}$ biologists study the ecological causes of individual differences in where fire salamanders (Salamandra salamandra) deposit their offspring. To do so, they describe the mechanism of niche choice, investigating how specific entities, such as female salamanders, larvae, and parasites, and activities, such as mating and growing, work together to cause the individual differences in maternal choice of offspring developmental environment.

$\mathrm{NC}^{3}$ mechanisms are an instance of what has been called "individual-level mechanisms" (Pâslaru 2018, 359). This is because they operate at the level of individual organisms and their abiotic and biotic environment, such as fire salamanders and their depositing larvae behavior. By contrast, the third abstract question is about genetic, transcriptomic, epigenetic, hormonal, and other physiological mechanisms. These mechanisms "underlie" individual differences because their componentsgenes, genomes, hormones, and other physiological processes-are located on a lower level of organization than individual organisms. Hence, $\mathrm{NC}^{3}$ mechanisms and molecular mechanisms underlying individual differences must be kept apart (Kaiser and Trappes forthc.).

\footnotetext{
5 Projects in the CRC are labelled with a letter (A-D) and two-digit number. For clarity, we also include the PI's name in brackets.
} 


\section{The CRC's specific research questions}

None of the individual projects in the CRC directly addresses abstract questions about causes, consequences, and underlying mechanisms of individual differences. Researchers study single species in laboratory or field conditions using observation, experiments, or modelling. Here we provide some examples to show that the CRC's abstract questions are addressed by answering specific questions, which are usually about finding out a restricted number of causes, consequences, or underlying mechanisms (or even just parts of them).

The first two abstract questions are often addressed together by studying specific cases of $\mathrm{NC}^{3}$ mechanisms as well as the fitness consequences of individual differences. For instance, Project A03 (Schielzeth) studies the way steppe grasshoppers (Chorthippus dorsatus) jump to escape predators. Juveniles are raised in either bare cages or cages with various objects inside, such as grass and sand. The complexity of the environment experienced by juveniles is treated as a possible ecologicaldevelopmental cause of individual differences in the grasshoppers' jumping behavior, a case of niche conformance (i.e., individuals adjust their phenotype differently to different environments). The same project uses computer simulations to study evolutionary causes and consequences of individual differences. The simulations test whether and how individual differences in jumping behavior can be maintained in a population in response to a predator.

Another project that addresses the first two abstract questions is Project $\mathrm{C} 03$ (Krüger). This project looks at where common buzzards (Buteo buteo) choose to construct their nests (niche choice) and what kinds of greenery they use to decorate them (niche construction). First, the project looks at whether individual differences in nesting behaviors are caused by the nest an adult experienced when they were a nestling; this is a developmental cause. A second question is how changes in ecological communities, such as the arrival of the eagle owl (Bubo bubo), which preys on buzzards, affect individual differences in nest location and decoration; this is an ecological cause. Third, the project looks at the fitness consequences of individual differences in nesting behavior and thus asks about the evolutionary consequences of these individual differences.

The third abstract question is usually addressed by targeting specific genetic, transcriptomic, epigenetic, hormonal, and other physiological mechanisms that underlie individual differences and changes in the phenotypic traits and niches of individuals. For instance, Project C04 (Gadau) looks at what mechanisms might underlie individual differences in the amount of aggression and prosocial behavior shown by harvester ant queens (Pogonomyrmex californicus). The project tests whether there are correlations between aggression and genetic and epigenetic differences, such as different alleles, DNA methylation, or small non-coding RNA. It also targets a hormonal factor (juvenile hormone titer) as well as the metabolic rate as possible physiological mechanisms for aggressive behavior.

Another project that studies underlying mechanisms is Project A02 (Richter and Sachser). This project studies optimistic and pessimistic personalities in mice (Mus musculus), looking at why some individuals consistently judge an ambiguous stimulus to be positive, and others consistently judge it to be negative. One of the central 
questions is whether the epigenetic modification of certain candidate genes underlies the observed behavioral differences in optimism and pessimism.

\section{Individual differences and biological individuality}

The biologists in the CRC are generally not concerned with how to identify individuals, nor with defining their boundaries, parts, and unity. To them it is largely obvious how to pick out and count the various organisms they study (e.g., mice, grasshoppers, buzzards, beetles, seals, and salamanders). Only one project studies an organism-harvester ants-that belongs to the class of problem cases that philosophers discuss (recall "Origins of the problematic nature of biological individuality" Section). Even in this project it seems obvious to the biologists that ants-not ant colonies - are the proper biological individuals to study.

Rather than asking 'What is a biological individual?' or 'Which objects are biological individuals?', the CRC asks, 'How do biological individuals differ from each other, how does this come about, and what are the consequences?' The focus is thus not on identifying biological individuals and distinguishing them from nonbiological individuals. The CRC also does not study how different kinds of biological individuals, such as evolutionary individuals, developmental individuals, and so on, diverge. Instead, the CRC looks into the set of all biological individuals and asks how we can distinguish one biological individual from another. For example, Project C04 (Gadau) studies how ant queens differ in their aggression level, and Project A02 (Richter and Sachser) investigates individual differences in the personalities of mice.

Research into individual differences, including the research in the $\mathrm{CRC}$, treats individual differences as effectively synonymous with individuality. "Behavioral individuality," for instance, is used to refer to behaviors that differ between individuals, and especially to differences that are persistent or change in some specific way (Barash 1997; Freund et al. 2013; Vogt 2015; Bierbach et al. 2017). Individual specialization is often seen as a way that individuality appears in ecological models, and individualized or individual niches are the consequence of such specialization (Dall et al. 2012; Fodrie et al. 2015). Thus, when biologists investigate individual differences, they often describe themselves as investigating individuality. This is also why the main goal described in the title of the CRC is the provision of a "novel synthesis of individualization across behavior, ecology and evolution."

One might object that the biologists in the CRC are just using the term 'individuality' with a meaning distinct from the meaning of 'individuality' in the philosophical debate about biological individuality. However, questionnaires and interviews we have conducted (Trappes 2021) suggest that this is not the case and that the biologists' way of reasoning about individuality in terms of individual differences bears close relations to philosophical discussions about biological individuality. For instance, when asked what about their research organisms makes them individuals, the biologists list classical criteria of biological individuality (e.g., being a unit of selection, undergoing reproduction, having a metabolism, being independent from the environment) alongside criteria concerning individual differences (e.g., personality, singularity, distinctness from others, uniqueness). This supports the thesis that 
philosophers of biology and the biologists in the CRC mean similar things when they talk about (biological) individuality.

In the next two sections we further substantiate this claim. We explicate two sets of philosophical questions that arise from the CRC's studies of individual differences-questions about uniqueness and questions about temporality (including change and temporal stability) — and we argue that these questions belong to the problem agenda of biological individuality.

\section{Uniqueness questions}

In this section we consider how questions about uniqueness emerge from the CRC's studies on individual differences, arguing that uniqueness questions should be recognized as central questions in the problem agenda of biological individuality. We begin by considering the links between individual differences, uniqueness, and individuality in the CRC's research ("Individual differences, uniqueness and individuality in the CRC" Section). We then introduce the uniqueness questions that we think should be recognized as central questions in the problem agenda of biological individuality and consider how they relate to other questions in the problem agenda ("Asking about uniqueness in philosophy of biology" Section). Finally, we provide further support for including uniqueness questions in the problem agenda by drawing an analogy to general metaphysics ("Uniqueness and individuality in metaphysics" Section).

\section{Individual differences, uniqueness and individuality in the CRC}

Studying how and why individuals differ from each other can involve reasoning about what makes individuals unique or distinct from others. For instance, behavioral biologists sometimes look for individuals with "unique personalities" (Bell 2007, 540). Most empirical studies, however, focus on individual differences rather than on uniqueness.

That some individuals differ from each other with respect to a trait or trait value does not typically mean that the trait or trait value is unique: there may be several individuals that have the same trait or trait value. For instance, individual guinea pigs (Cavia aperea $f$. porcellus; B01, Kaiser and Sachser) differ in their social behavior, but the behavioral traits that are studied, such as being aggressive or dominant (or less or more dominant or aggressive than the average), are ascribed to more than one individual. Indeed, most of the experiments and empirical studies in the CRC group together individuals with similar traits and study, for instance, optimistic mice, aggressive ant queens, and zebra finch males with denser ejaculates. This grouping of individuals is necessary for many of the observational, experimental and statistical methods, and is particularly important for developing generalizations about the causes, consequences, and underlying mechanisms of individual differences.

Although biologists do not typically study unique traits, questions of uniqueness do arise in the context of research on individual differences. Biologists in the CRC 
bring up uniqueness in theoretical discussions, stating that individuals have unique sets of traits. The individual differences they study are elements of these unique sets; they contribute to uniqueness by adding differences between individuals. The theoretical discussions in the $\mathrm{CRC}$ as well as the questionnaire and interviews conducted with CRC members (Trappes 2021) demonstrate that the concept of uniqueness plays a central role in understanding and researching individual differences.

Questions about uniqueness, which arise when studying individual differences, in turn connect to biological individuality. Biologists in the CRC frequently explain or specify the concept of individuality and related concepts such as individualized phenotype and individualized niche in terms of uniqueness (Trappes 2021). In addition, we showed in "Individual differences and biological individuality" Section that biologists in the CRC understand the concept of individuality in a way closely connected to how biological individuality is discussed in the philosophical debate.

There is also strong empirical evidence that biological individuals are unique. The CRC's research provides many examples of how individuals differ from one another in many different traits. Genetically identical individuals such as monozygotic twins typically differ in at least one (and usually more than one) phenotypic trait (Wong et al. 2005). There is also evidence that differences arise amongst genetically identical individuals raised in the same environment (Vogt et al. 2008; Gärtner 2012; Freund et al. 2013; Vogt 2015; Bierbach et al. 2017). Given the high prevalence of differences between individuals in many different traits, there is strong empirical support for the inference that individuals, in general, will have unique sets of traits.

In sum, empirical facts about biological individuals and about conceptual and reasoning practices in the CRC suggest that uniqueness is an important aspect of biological individuality and that questions about uniqueness should be a central element of the problem agenda of biological individuality. It might be objected that the mere fact that biological individuals are unique does not show that uniqueness is a necessary condition for or constitutive dimension of biological individuality. Even the fact that biologists in their research practice assume a very close relation between biological individuality and uniqueness does not prove that uniqueness is necessary for biological individuality. This might be true, but we do not see the need to make such a strong claim.

Uniqueness might not be a necessary condition for (or a constitutive dimension of) biological individuality but it can still be an important aspect of biological individuality and thus should be included in the problem agenda of biological individuality. From a pluralistic perspective it seems plausible to assume that phenotypic uniqueness (see next section), is only one plausible criterion for identifying biological individuals, amongst many others, which is particularly relevant in fields such as behavioral ecology. Hence, uniqueness can be a central aspect of biological individuality without being necessary for it.

\section{Asking about uniqueness in philosophy of biology}

What, exactly, is the relation between uniqueness and biological individuality assumed in the CRC? In their research uniqueness is a feature ascribed to biological 
individuals due to their unique sets of phenotypic traits. Even though the biologists are not concerned with identifying biological individuals and distinguishing them from non-individuals (recall "Individual differences and biological individuality" Section), they implicitly provide an answer to the question "What is a biological individual?' The answer is 'Biological individuals are unique in the sense that they have unique sets of phenotypic traits.'

The assumed close connection between uniqueness and biological individuality gives rise to various philosophical questions, which can be subsumed under the general philosophical question 'In what way are biological individuals unique?' Different sets of more specific questions about uniqueness in relation to biological individuality can be distinguished. Not all of these uniqueness questions are new to the debate about biological individuality. But some of them are truly novel and all of them should be recognized and systematically addressed as central parts of the problem agenda of biological individuality.

First, what kinds of properties make biological individuals unique? The CRC focuses on studying individual differences in phenotypic traits, often in behavioral traits. This suggests that the uniqueness of biological individuals can be understood as phenotypic uniqueness (of which behavioral uniqueness is a major part). In addition, the idea of unique individualized niches seems to imply that ecological properties (e.g., social relations or resource use) also affect the uniqueness of a biological individual.

Furthermore, several other kinds of properties have been discussed by philosophers in relation to the uniqueness of biological individuals. The type of uniqueness that has received most attention so far is genetic uniqueness (Hull 1978; J Wilson 1999; Santelices 1999; De Sousa 2005; Clarke 2010, 317; 2012; Herron et al. 2013; Chauvier 2017). Since there are many biological individuals with nonunique genomes, genetic uniqueness is usually rejected as a criterion of biological individuality (Hull 1978; Santelices 1999; Hauskeller 2004; De Sousa 2005; Folse and Roughgarden 2010; Clarke 2012; Elwick 2017). Other kinds of uniqueness that have been discussed or mentioned include immunological uniqueness (Pradeu 2012; Minelli 2020), historical uniqueness (Hull 1978), epigenetic uniqueness (Boniolo and Testa 2012; Gorelick 2012) and phenotypic uniqueness (Hull 1978; De Sousa 2005; Guay and Pradeu 2015; Nyhart and Lidgard 2017). Most discussions focus on only one type of unique property (e.g., genetic uniqueness or phenotypic uniqueness), leaving little space for a general discussion about uniqueness and biological individuality. We think that these different strands of the debate should be drawn together and the question which kinds of properties determine the uniqueness of a biological individual should be systematically addressed.

Second and relatedly, we can ask if there is a priority of some kinds of properties over others in determining the uniqueness of a biological individual. For example, is it plausible to assume that only phenotypic traits determine the uniqueness of an individual and that other properties, such as genetic, epigenetic, or hormonal properties, are relevant only to the underlying mechanisms of individual differences (recall "The CRC's abstract research questions" Section)? Moreover, are behavioral traits more important than other phenotypic traits because they determine the personality of individual organisms (Kaiser and Müller 2021)? 
A third question concerns how uniqueness can help to answer other central questions in the problem agenda. Even though uniqueness questions are questions about biological individuality in their own right, they can also contribute to answering the question of how to identify biological individuals. For instance, the CRC's assumption that biological individuals have unique sets of phenotypic traits could give rise to a criterion for identifying biological individuals, which we call the criterion of phenotypic uniqueness. It can be formulated as follows:

\section{Phenotypic Uniqueness Criterion for Biological Individuality}

An object $x_{1}$, which is a member of the population $p$, is a biological individual if.

(1) $x_{1}$ differs in at least one phenotypic trait from some of the other members $x_{2}-x_{n}$ of $p$; and

(2) no other member of $p$ shares with $x_{1}$ the same set of phenotypic traits.

This criterion can identify what could be called phenotypic individuals (with "behavioural individuals" as a subtype; Smith-Ferguson and Beekman 2019).

Some philosophers, such as David Hull, have rejected phenotypic uniqueness as irrelevant to biological individuality, chiefly because we can imagine two phenotypically identical individuals that are nevertheless distinct (Hull 1978, 345). On the other hand, a phenotypic uniqueness criterion has been supported by behavioral ecologists as suitable for individuating "non-standard" organisms such as social insects and slime molds (Smith-Ferguson and Beekman 2019). Given this disagreement, it makes sense for philosophers to ask, 'Is phenotypic uniqueness a plausible criterion of biological individuality?'.

We think that the phenotypic uniqueness criterion could help to identify one kind of biological individual among others. Thus, although many individual organisms satisfy the phenotypic uniqueness criterion, it need not be applicable to all biological individuals, nor can it account for all different practices of identifying biological individuals in different research contexts. Formulating such a criterion of phenotypic uniqueness is hence compatible with the pluralistic view that there are various types of biological individuals identified on the basis of different individuality concepts and criteria.

A final question about uniqueness we would like to raise is whether biological individuals could be unique in the different ways in which they satisfy certain criteria or realize types of biological individuality. ${ }^{6}$ According to this line of reasoning, different biological individuals could have, for instance, unique modes of cohesion, differentiation, autonomy, or reproduction. The way in which the amoebae of a multicellular slime mold slug (Dictyostelium discoideum) form a cohesive whole, for example, is very different from the type of cohesion that exists between the different cells of a grasshopper or a buzzard. Likewise, the green

\footnotetext{
${ }^{6}$ We thank the editors of Biology and Philosophy for this idea.
} 
Box 1 Uniqueness questions

General uniqueness question:

In what way are biological individuals unique?

Specific uniqueness questions:

(1) What kinds of properties make biological individuals unique? Are only phenotypic traits relevant or do also other kinds of properties (e.g., genetic, epigenetic, historical, or immunological properties) determine the uniqueness of a biological individual? Does the idea of unique individualized niches imply that ecological properties, too, express the uniqueness of a biological individual?

(2) Is there a priority of some kinds of uniqueness properties over others? For instance, are behavioral traits more important to the uniqueness of a biological individual than other phenotypic traits?

(3) How can the uniqueness of biological individuals help to identify them (i.e., answer the identification question)? Is phenotypic uniqueness a plausible criterion of biological individuality?

(4) How do biological individuals (of different species) realize types of biological individuality differently? How can these unique modes of, for instance, cohesion, differentiation, autonomy, or reproduction be specified?

algae in a lichen possess a very different type of autonomy than that of the ants in an ant colony. Hence, individuals of many species can be said to be unique in the way they are biological individuals, that is, how they realize a certain kind of biological individuality and meet certain identification criteria.

In most cases the ways in which individuals realize a certain type of biological individuality will be the same for all members of a species. Hence, this way of thinking about what makes biological individuals unique places uniqueness on the species level. By contrast, conceiving of uniqueness as a feature of biological individuals locates uniqueness on the individual level, such that individuals differ in their phenotypic, genetic, or immunological properties. In our view, both ways to think about uniqueness - in terms of unique properties of individuals and unique ways to realize biological individuality-raise interesting philosophical questions about uniqueness that should be recognized as central parts of the problem agenda of biological individuality.

BOX 1 summarizes some different uniqueness questions that arise in relation to biological individuality and that can be subsumed under the general question 'In what way are biological individuals unique?'.

The third and fourth sorts of uniqueness questions indicate how closely connected some uniqueness questions are to the existing problem agenda of biological individuality (and its questions of identification, demarcation, parthood, and unity). Recognizing uniqueness questions as an independent, central part of the problem agenda of biological individuality contributes to the existing debate in a number of ways.

First, it broadens the scope of uniqueness considered by philosophers of biology. Rather than primarily looking at genetic uniqueness, and occasionally and separately at other sorts of unique properties, we can collect investigations about uniqueness under a central question. This will connect scattered debates and also draw attention to relatively overlooked sorts of uniqueness. For instance, philosophers could investigate behavioral uniqueness and uniqueness of ecological 
properties and consider how these sorts of uniqueness relate to genetic and epigenetic uniqueness.

Second, uniqueness questions bear interesting connections to existing questions in the problem agenda of biological individuality. Some connections are already clear from the existing use of uniqueness to answer questions about demarcation and identification. Exploring different types of uniqueness, rather than just genetic uniqueness, might contribute to new ideas about how to answer such questions. We have already discussed the potential for new criteria for identifying biological individuals, such as the phenotypic uniqueness criterion. Another example is the role of uniqueness for demarcation. Although genetic properties cannot be used to demarcate clones or identical twins, it is often possible to use unique epigenetic or immunological properties to answer demarcation questions about genetically identical individuals (Boniolo and Testa 2012; Pradeu 2012). In addition, unique behavioral or even ecological properties could be used to demarcate individuals in a comprehensive and systematic way, without requiring molecular investigation of the candidate individuals. Asking uniqueness questions can also connect to other questions about biological individuality by introducing new considerations about the existing ways we have to answer such questions, such as whether there might be unique ways to satisfy criteria of biological individuality.

\section{Uniqueness and individuality in metaphysics}

In the previous two subsections we have argued that questions about uniqueness arise in relation to biological individuality in biological practice and that they are closely connected to existing discussions in the philosophical problem agenda of biological individuality. A third and final argument for including uniqueness questions as central questions in the problem agenda of biological individuality relies on an analogy to general metaphysics. In metaphysics, unique identity is often considered to be an important aspect of individuality. Philosophical debate centers on which properties are unique and can thus determine identity for individuals. By analogy, we think that philosophers of biology should also conceive of uniqueness as a central aspect of biological individuality.

Many metaphysicians argue that individuality consists of two aspects: countability and identity. ${ }^{7}$ For instance, Jonathan Lowe states that "what 'individuates' an object [...] is whatever it is that makes it the single object that it is-whatever it is that makes it one object, distinct from others, and the very object that it is as opposed to any other thing" (Lowe 2003, 75; emphasis in original). Accordingly, Lowe provides the following definition of an individual: "Something, $x$, is an individual if and only if (1) $x$ determinately counts as one entity and (2) $x$ has a determinate identity." (Lowe 2016, 50; emphasis in original) Lowe argues that these two requirements for individuality must be kept apart because concepts of countability

\footnotetext{
7 Terms used to describe individuality are often confusing. Singularity, for instance, can refer at once to being a single entity and being unique. For clarity's sake, we will refer primarily to countability and identity.
} 
and identity are not necessarily always co-applicable. ${ }^{8}$ Other metaphysicians identify the same two requirements on individuality: "By an individual we understand something that is considered as one [...] and which can be reidentified in a different situation [...] as being that same item." (Krause and Arenhardt 2016, 62).

How does identity relate to uniqueness? According to Leibniz's Law, two entities that share all the same properties must be one and the same entity (Forrest 2016). It follows that two distinct entities cannot share all the same properties: there must be some properties that are not shared with any other entity, that is, unique properties. There is a long debate about which properties could serve this role; essences or haecceities, qualitative properties, and relational properties such as spatiotemporal position have all been considered as candidates for defining identity (Strawson 1959; Hawley 2009). ${ }^{9}$ Typically, the aim is to identify which properties are metaphysically necessarily unique, that is, unique in all possible worlds or all conceivable situations (Hawley 2009). ${ }^{10}$

So, when asking questions about individuality, many metaphysicians hold that we must also consider identity and hence ask questions about what makes individuals unique. What does that mean for philosophers of biology? Metaphysicians focus on individuality and identity in the most general sense, whereas philosophers of biology are only concerned with the individuality of biological entities. But if unique identity is often seen as a constitutive aspect of individuality in general, then it is plausible to assume that it could play a similar role for biological individuality. Hence, we suggest that philosophers of biology should also be asking questions about uniqueness-not just in the service of other questions about biological individuality, but as questions in their own right.

The questions about uniqueness (and identity) asked by metaphysicians and philosophers of biology will likely differ. First, they may focus on different properties. While metaphysicians ask about general properties, such as spatiotemporal position or essences, philosophers of biology focus on biological properties, such as phenotypic, genetic, or epigenetic properties. Second, philosophers of biology need not focus on metaphysical necessity; contingency or even some form of biological necessity may be adequate for investigating the uniqueness of biological individuals. Third, the uniqueness about which philosophers of biology ask comes in degrees; some individuals can be more unique than others, for instance if they share fewer phenotypic properties with other individuals. In contrast, metaphysicians are often

\footnotetext{
${ }^{8}$ For example, the water in my bathtub has a determinate identity without a determinate countability, whereas the orbital electrons of a helium atom are clearly two electrons but they do not have a determinate identity because there is no fact of the matter as to which electron is spin-up and which is spin-down (Lowe 2016, 50-53).

${ }^{9}$ Note that the connection between identity and individuality should hold regardless of which sorts of properties one thinks are identity-determining. In particular, it is not necessary to believe, as Lowe does, that individuals have essences to nevertheless believe that identity is a crucial aspect to individuality.

${ }^{10}$ The metaphysical debate about identity involves more than just questions about uniqueness. Another topic is persistence, or what determines identity over time. Answers to questions about persistence often involve more than just uniqueness, including for instance some sort of temporal continuity or unity between temporal parts. See "Temporal stability and biological individuality" Section, where we briefly raise such considerations.
} 
(though not always) interested in absolute rather than relative identity. They therefore ask about which properties are unique simpliciter; an object being unique in more properties is irrelevant to its being identical with itself.

Finally, philosophers of biology will not always be addressing the same sorts of metaphysical and conceptual problems as metaphysicians. Metaphysicians often debate problem cases of identity, such as whether there could be two qualitatively identical but numerically distinct objects. Since biological individuals typically have unique spatiotemporal positions, hard identity problems do not usually arise, and asking about biological uniqueness is not usually required to decide whether two individuals are distinct. Nevertheless, uniqueness of biological properties might still be sufficient to solve some identity problems. For instance, as we mentioned in "Asking about uniqueness in philosophy of biology" Section, it is possible to distinguish individuals based on phenotypic uniqueness rather than only spatiotemporal distinctness. In addition, philosophers of biology might use questions about the uniqueness of biological individuals to address other issues related to biological individuality. We have already discussed the idea of uniqueness criteria for identifying biological individuals. In addition, the uniqueness of biological properties contributes to the discussion about why biological individuality may come in degrees (e.g., Pepper and Herron 2008; Godfrey-Smith 2009; Clarke 2012; Pradeu 2016) by providing an instance of measurable degrees of individuality based on the number of properties that are unique.

Drawing an analogy between general metaphysics and philosophy of biology is possible despite these differences because they also possess important similarities. For instance, they analyze parallel phenomena of individuality in general and biological individuality, and they share the structure of the questions about which sorts of properties are unique. In addition to supporting our argument for explicitly including uniqueness questions in the problem agenda of biological individuality, we hope the analogy will also facilitate the use of conceptual resources from the long metaphysical tradition by philosophers of biology.

We began this section by showing how the CRC's questions about individual differences lead to philosophical questions about what makes biological individuals unique. We now have several reasons for why such questions belong to the problem agenda of biological individuality. Uniqueness and individuality are strongly connected in biological research, in philosophy of biology and in general metaphysics, indicating that questions about uniqueness should be explicitly acknowledged as part of the problem agenda of biological individuality. Doing so will help to systematize and broaden the existing scattered references to uniqueness in the literature on biological individuality.

\section{Temporality questions}

In addition to questions about uniqueness, the study of individual differences in the CRC raises two related sets of philosophical questions about temporality: one about kinds of changes in individuality at various timescales ("Changes in biological individuality over various timescales" Section) and the other about temporal stability 
("Temporal stability and biological individuality" Section). Both sets of questions have precedent in discussions about temporal aspects of biological individuality, but are often underrecognized. Like questions about uniqueness, questions about temporality should be explicitly included into the problem agenda of biological individuality.

\section{Changes in biological individuality over various timescales}

One main philosophical question about temporality that arises from the CRC's research questions is about what kinds of changes biological individuality can undergo at various temporal scales.

Many of the projects in the CRC study how individual differences arise and change at evolutionary, ecological, and developmental timescales. At the evolutionary scale, for instance, Project D03 (Wittmann) uses computer modelling to look at how individual differences in genotype, plasticity, and reaction norms arise and change over thousands of generations. At the ecological scale, Project C03 (Krüger) studies changes in where and how buzzards build their nests over successive breeding years and over several generations. At the developmental timescale, Project B01 (Kaiser and Sachser) looks at how guinea pigs' social behavior changes during adolescence and adulthood (see Sachser et al. 2018). Studying changes in individual differences at different timescales fits into the abstract questions about causes and consequences of individual differences and is hence a major part of the CRC's research.

In "Uniqueness questions" Section we showed that the biologists' questions about individual differences give rise to philosophical questions about uniqueness. Since the CRC also studies how individual differences change over time, the philosophical question arises about how uniqueness changes at different timescales and how this affects biological individuality. When individual differences are generated or cease to exist, this may alter which types of or how many properties are unique for an individual. Individuals differ in some respects and not in others, and changes in their traits can lead to an increase or decrease of both the number of traits that an individual shares with other individuals and the number of other individuals with which the individual shares a trait. Accordingly, changes in the traits of an individual can make the individual more or less unique and thus increase or decrease its degree of individuality. Hence, the CRC's studies about how individual differences change not only give rise to the question what kinds of changes uniqueness and biological individuality can undergo, they also provoke more specific questions about degrees of uniqueness and biological individuality and how these degrees can change at different timescales.

Posing the temporality question 'What kinds of changes can biological individuality undergo at what timescales?', as such, is not new. Because this question encompasses more than just changes in uniqueness, any changes in biological individuality can be (and partly have been) investigated in answer to this question. This includes changes in whether and how certain individuality criteria are satisfied, changes in degrees of biological individuality, and changes in different kinds of biological individuality. 
The existing philosophical debate on biological individuality has considered changes in kinds and degrees of biological individuality at an evolutionary scale: so-called evolutionary transitions in individuality. This research focuses on the evolution of complex biological individuals exhibiting functional integration and reproductive specialization, often considered as criteria for biological individuality (e.g., Buss 1987; Maynard Smith and Szathmary 1995; Godfrey-Smith 2009; Calcott and Sterelny 2011). ${ }^{11}$ The questions asked in this discussion include "How did unicellular organisms give rise to multicellular organisms? How did some multicellular animals come to form superorganisms?" (Love and Brigandt 2017, 325). Such transitions represent significant changes in biological individuality occurring on the macro-evolutionary scale.

In addition, a few philosophers have asked about developmental changes in biological individuality. James Griesemer (2018), for example, has proposed that different degrees of individuality are exhibited at different developmental stages. Similar thoughts have come from those studying complex life forms, such as algae and amoebae which form temporary colonies, thus exhibiting changes in the degree of individuality over shorter timescales (Herron et al. 2013). Earlier philosophers of biology, such as Georges Canguilhem (1991; Gayon 1998) and Gilbert Simondon (1992), also discussed changes in biological individuality through development. Nevertheless, such work is an exception. Indeed, a common unstated assumption is that biological individuality is constant across the lifespan of an individual; while the individual may change during its lifetime, its status as an individual and its degree of individuality does not. ${ }^{12}$

The changes that individual differences and uniqueness can undergo invite us to consider changes in biological individuality at all time scales. Individual differences can change over a smaller number of generations due to micro-evolutionary processes, and through developmental and ecological processes over just a few generations or even within a single generation. We can therefore start to ask about how biological individuality changes not just during macro-evolution, but also at microevolutionary, ecological, and developmental timescales. Asking these questions systematizes discussions about macro-evolutionary and developmental changes in biological individuality into a clear set of questions within the problem agenda of biological individuality. In addition, if we think about individuality in terms of individual differences and uniqueness, it will become clear that change in individuality

\footnotetext{
11 Much of this literature also looks at the role of inheritance mechanisms for biological individuality (McConwell 2017). Note however that inheritance mechanisms are not mechanisms for the changes in biological individuality observed over evolution; instead, evolutionary changes in individuality are produced by changing the inheritance mechanisms. In contrast, the mechanisms studied in the CRC are mechanisms involved in producing and changing individual differences.

12 This assumption may be attributable to the focus on evolutionary individuality, which often involves identifying bearers of fitness (Clarke 2013). Since fitness is usually assigned to an entire life rather than to life stages, so too is evolutionary individuality. Hence, biological individuality is thought not to change at developmental timescales, and the focus is rather on determining the temporal boundaries of an individual, that is, when an individual begins and ends (Maienschein 2011; Minelli 2011; GodfreySmith 2015), or on the persistence conditions that allow an individual to continue to exist despite radical changes during development (Hull 1978; Pradeu 2018).
} 
is not restricted to the rise and decay of individuality, such as multicellular organisms evolving through evolutionary transitions, or a slime mold emerging from a set of solitary amoebae once they are spatially and functionally organized in a specific way. Instead, change in individuality in terms of individual differences includes gradual changes in degrees of individuality.

\section{Temporal stability and biological individuality}

The second set of questions about temporality generated by the CRC's research concerns temporal stability. The main question is how much and which kind of temporal stability is needed for biological individuality. Contrary to questions about changes in biological individuality ("Changes in biological individuality over various timescales" Section), questions about temporal stability have not been directly addressed in the debate about biological individuality so far-though we show that they can be fruitfully related to existing debates about criteria of biological individuality and the persistence of biological individuals.

When asking about individual differences biologists look for a certain kind of temporal stability in phenotypic traits. Two subtypes of temporal stability can be distinguished: continuity and repeatability. Continuous individual differences are those that are constantly exhibited over an extended period of time, such as a whole developmental period or a whole lifetime. For instance, color pattern is an important continuous individual difference in adult fire salamanders. Project A04 (Caspers) seeks to determine how larval experience affects this continuous individual difference in adulthood. Repeatability, by contrast, refers to traits that are exhibited consistently by an individual at many distinct time points (Bell et al. 2009; Novak and Tinker 2015). Animal personalities, for instance, are individual differences in behavioral phenotypes that are stable across times and contexts (Wolf and Weissing 2012, 453). For example, an individual beetle is characterized as bold only if it shows the same bold behavior in repeated behavioral tests (Tremmel and Müller 2013). In general, biologists discuss whether relevant individual differences should extend across developmental periods (Sih et al. 2004), at what timescales they exist and can be studied (Fodrie et al. 2015; Novak and Tinker 2015), and how continuous or repeatable a phenotypic trait must be in order to count as a relevant individual difference (Bell et al. 2009).

This is how biologists investigate and reason about the temporal stability of individual differences. In our view, this shows that certain amounts and kinds of temporal stability of phenotypic traits are required for the uniqueness of biological individuals. The biologists' research questions and assumptions thus generate philosophical questions about temporal stability and biological individuality. The central question is 'How much and what kind of temporal stability is needed for biological individuality?' This question invites further, more specific questions: How is temporal stability quantified and how much temporal stability is sufficient for biological individuality? What should be temporally stable (e.g., which kinds of phenotypic traits or properties)? Are the two kinds of temporal stability — continuity and repeatability—equally important to biological individuality? 
Box 2 Temporality questions

General temporality question 1 :

What kinds of changes can biological individuality undergo at what timescales? Specific temporality questions 1 :

(1) How did unicellular organisms give rise to multicellular organisms? How did some multicellu-

lar animals come to form superorganisms?

(2) How does the degree of biological individuality change during development?

(3) How does individuality change at micro-evolutionary and ecological timescales?

(4) Do changes in individual differences imply gradual changes in degrees of individuality?

General temporality question 2 :

How much and what kind of temporal stability is needed for biological individuality?

Specific temporality questions 2 :

(1) How much temporal stability is sufficient for biological individuality?

(2) What should be temporally stable (e.g., which kinds of phenotypic traits or properties)?

(3) Are the two kinds of temporal stability — continuity and repeatability — equally important to biological individuality?

(4) What would a processual account of identity over time look like?

(5) What changes does biological individuality require?

Asking questions about the temporal stability of biological individuality connects to discussions of the persistence conditions of biological individuality (e.g., Guay and Pradeu 2015; Dupré 2018) and hence may lead to new insights concerning questions of identification, demarcation, parthood, and unity. For instance, in the debate about biological individuals as processual, it has been proposed that processes can be identified by their continuity over time (Guay and Pradeu 2015). On the other hand, paying attention to repeatability may indicate that a biological individual can persist without it continuously exhibiting the features that satisfy a criterion of biological individuality. For instance, an organism that is repeatedly but not constantly autonomous could thereby remain a biological individual. Establishing when and at what point repetition and continuity qualify an organism for individuality consequently presents itself as an important task for philosophers of biology.

Asking about temporal stability also sheds light on existing criteria of biological individuality. As we discussed in "Changes in biological individuality over various timescales" Section, philosophers often assume that individuality does not change at the developmental timescale, which means the criteria they choose should ensure that individuality is temporally stable. For instance, criteria such as cohesion or functional-causal integration, as well as functional or metabolic autonomy or discreteness, ensure the stability of an individual over time in response to changes in the environment. Other criteria for identifying biological individuals also presuppose a certain kind of stability, such as genetic homogeneity, a stable succession of the stages of a life cycle, or being a stable unit on which natural selection acts. Of course, most criteria assume that certain kinds of changes are also crucial to individuality (Lidgard and Nyhart 2017, 19-21). For example, metabolic autonomy requires metabolic processes such as the conversion of food to energy (Dupré 2018), units of selection require inheritance mechanisms (Godfrey-Smith 2009; McConwell 2017), and an immunological criterion requires immunological processes for demarcating biological individuals (Pradeu 2012). Nevertheless, it is typically a temporally stable 
The Extended Problem Agenda of Biological Individuality

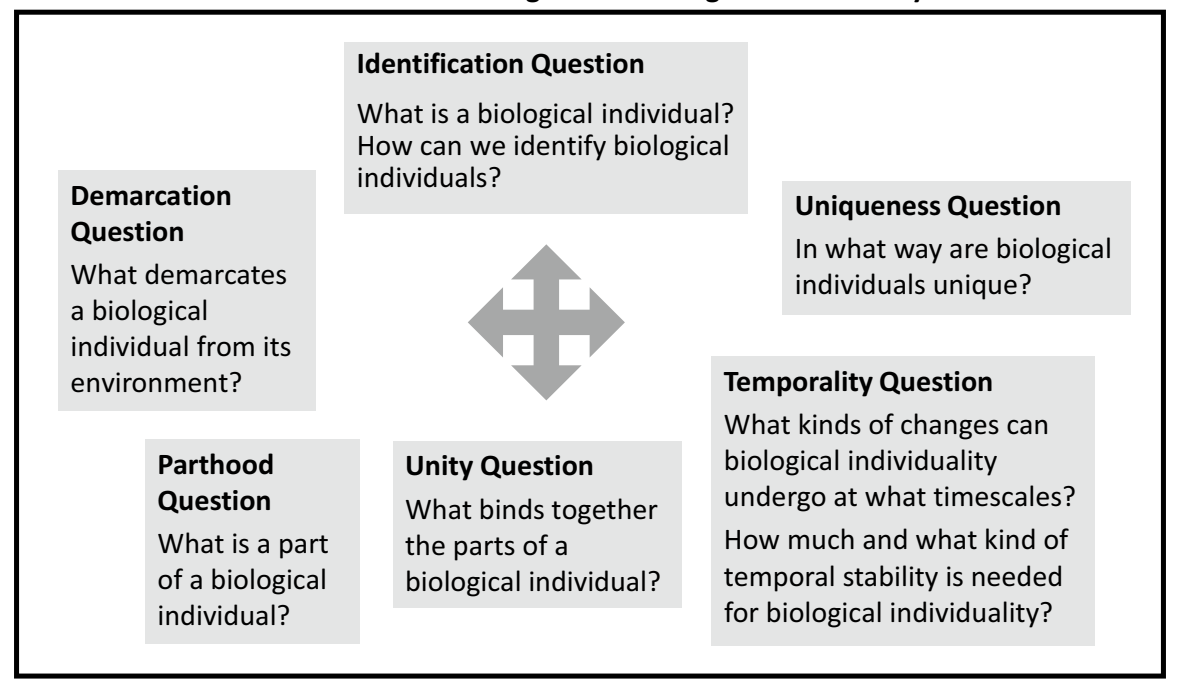

Fig. 2 The Extended Problem Agenda of Biological Individuality. Central questions in the problem agenda, now including uniqueness and temporality questions, are linked by various interrelations, indicated by the multi-headed arrow

feature, such as the reoccurrence or continuation of a certain change, that serves as a criterion for biological individuality.

In BOX 2 we summarize the temporality questions. Like questions about uniqueness, questions about temporality that arise from the CRC's research are connected to prominent questions in the problem agenda of biological individuality, help to organize and systematize existing discussions, and generate distinct insights about biological individuality. Hence, the two sets of temporality questions should be explicitly included into the problem agenda of biological individuality.

\section{Conclusions}

The problem agenda of biological individuality has often concentrated on questions about identification, demarcation, parthood, and unity. By studying a contemporary example of biological practice in an interdisciplinary research group from ecology, evolutionary biology, and behavioral biology, we have revealed two underestimated sorts of questions about biological individuality: uniqueness and temporality questions. We have argued that these questions should be included as explicit and central parts of the problem agenda of biological individuality, resulting in the extended problem agenda shown in Fig. 2.

In arguing for the explicit inclusion of uniqueness and temporality questions, we showed how they arise out of the CRC's research into individual differences and why they are questions about biological individuality. Uniqueness questions, such as 'In what way are biological individuals unique?', are generated 
based on questions about how biological individuals differ from one another in sets of phenotypic traits and other properties. We demonstrated the connections between uniqueness and biological individuality in biological research and the existing philosophical debate and supported this with an analogy to metaphysical theorizing about individuality, identity, and uniqueness. Asking questions about uniqueness will bring together scattered discussions of various types of uniqueness and stimulate a systematic discussion of uniqueness in relation to biological individuality.

The first major temporality question, 'What kinds of changes can biological individuality undergo at what timescales?', arises out of the CRC's abstract research questions about causes, consequences, and mechanisms of individual differences. Asking how biological individuality changes organizes and expands the existing debate on changes in individuality to include changes at all temporal scales and involving both kinds and degrees of individuality. The second major temporality question, 'How much and what kind of temporal stability is needed for biological individuality?', takes its cue from the requirement for temporal stability in the study of individual differences. This question opens up a new topic for philosophical research and promises to shed light on existing ideas about persistence and identification.

Acknowledging the uniqueness and temporality questions as explicit and central parts of the problem agenda of biological individuality does increase its complexity. But it does so at a significant benefit to the scope and organization of the debate, the connections it makes to other fields, and its proximity to biological practice. First, asking about uniqueness and temporality brings underrecognized aspects of biological individuality and scattered existing discussions into coherent and comprehensive research agendas for philosophy. This may also affect how existing questions about identification, parthood, demarcation, and unity are answered. Second, drawing the connection to metaphysics provides new tools and will hopefully lead to a fruitful dialogue. Finally, recognizing uniqueness and temporality questions as central parts of the problem agenda brings it further in line with areas of biological research in which individuality is discussed outside of its relation to problems of identification, such as the research into individual differences in behavior, evolution, and ecology that we investigated here. Hence, we believe that further exploration of the questions raised in this paper will lead to new insights about biological individuality and enhance the connection between metaphysics, philosophy of biology, and biological practice.

Acknowledgements This paper presents research that has been carried out in the project D02 "The Ontological Status of Individualised Niches" (396781820) of the SFB TRR 212 "A Novel Synthesis of Individualisation Across Behavior, Ecology and Evolution: Niche Choice, Niche Conformance, Niche Construction $\left(\mathrm{NC}^{3}\right)$ " funded by the German Research Foundation (DFG). We would like to thank all members of the SFB for their insights into their work and for their feedback to our ideas. Special thanks go to Sylvia Kaiser, Ulrich Krohs, Oliver Krüger, David Lambert, Behzad Nematipour, Norbert Sachser and Holger Schielzeth for their helpful comments on earlier versions of this paper. We also received fruitful feedback from the members of the graduate school (GRK 2073), the Research Seminar Theoretical Philosophy and the PhilBio Team of Bielefeld University, the participants of the book symposium on biological individuality at the ISHPSSB conference 2019 in Oslo, two anonymous reviewers and the editorial team of this journal. 
Funding Open Access funding enabled and organized by Project DEAL. This research was funded by the German Research Foundation (DFG) as part of the SFB TRR 212 (NC ${ }^{3}$ ) - Project number 316099922.

Open Access This article is licensed under a Creative Commons Attribution 4.0 International License, which permits use, sharing, adaptation, distribution and reproduction in any medium or format, as long as you give appropriate credit to the original author(s) and the source, provide a link to the Creative Commons licence, and indicate if changes were made. The images or other third party material in this article are included in the article's Creative Commons licence, unless indicated otherwise in a credit line to the material. If material is not included in the article's Creative Commons licence and your intended use is not permitted by statutory regulation or exceeds the permitted use, you will need to obtain permission directly from the copyright holder. To view a copy of this licence, visit http://creativecommons.org/licen ses/by/4.0/.

\section{References}

Araújo MS, Bolnick DI, Layman CA (2011) The ecological causes of individual specialisation. Ecol Lett 14(9):948-958

Barash DP (1997) In search of behavioral individuality. Hum Nat 8(2):153-169

Bell AM (2007) Animal personalities. Nature 447:539-540

Bell AM, Hankison SJ, Laskowski KL (2009) The repeatability of behaviour: a meta-analysis. Anim Behav 77(4):771-783

Bierbach D, Laskowski KL, Wolf M (2017) Behavioural individuality in clonal fish arises despite nearidentical rearing conditions. Nat commun 8:15361

Bolnick DI, Svanbäck R, Fordyce JA, Yang LH, Davis JM, Hulsey CD et al (2003) The ecology of individuals: incidence and implications of individual specialization. Am Nat 161:1-28

Boniolo G, Testa G (2012) The identity of living beings, epigenetics, and the modesty of philosophy. Erkenn 76:279-298

Bouchard F, Huneman P (2013) Introduction. In: Bouchard F, Huneman P (eds) From Groups to Individuals. MIT Press, Cambridge, Evolution and Emerging Individuality, pp 1-14

Boulter S (2013) Metaphysics from a Biological Point of View. Palgrave Macmillan, London

Bueno O, Chen R-L, Fagan MB (2018) Individuation, process and scientific practices. In: Bueno O, Chen R-L, Fagan MB (eds) Individuation, process and scientific practices. OUP, Oxford, pp 1-18

Buss L (1987) The evolution of individuality. Princeton University Press, Princeton

Calcott B, Sterelny K (2011) The major transitions in evolution revisited. MIT Press, Cambridge

Canguilhem G (1991) The normal and the pathological. Cohen R (trans), Zone Books, New York, Fawcett $\mathrm{C}$

Chauvier S (2017) Individuality and aggregativity. PTPBio, 9 (11).

Clarke E (2010) The problem of biological individuality. Biol Theory 5:312-325

Clarke E (2012) Plant Individuality: a solution to the Demographer's Dilemma. Biol Philos 27(3):321-361

Clarke E (2013) The multiple realizability of biological individuals. J Philos 110(8):413-435

Clarke E, Okasha S (2013) Species and organisms: What are the problems? In: Bouchard F, Huneman P (eds) From groups to individuals. evolution and emerging individuality. MIT Press, Cambridge, pp 55-73

Dall SRX, Bell AM, Bolnick DI, Ratnieks FLW (2012) An evolutionary ecology of individual differences. Ecol Lett 15:1189-1198

de Sousa R (2005) Biological individuality. Croatian journal of philosophy 5(2):195-218

Dupré J (2018) Processes, organisms, kinds, and the inevitability of pluralism. In: Bueno O, Chen RL, Fagan MB (eds) Individuation, Process, and Scientific Practices. OUP, New York, pp 21-39

Elwick J (2017) Distrust that particular intuition: resilient essentialisms and empirical challenges in the history of biological individuality. In: Lidgard S, Nyhart LK (eds) Biological Individuality Integrating Scientific, Philosophical and Historical Perspectives. University of Chicago Press, Chicago, pp 277-296 
Fodrie FJ, Yeager LA, Grabowski JH, Layman CA, Sherwood GD, Kenworthy MD (2015) Measuring individuality in habitat use across complex landscapes: approaches, constraints, and implications for assessing resource specialization. Oecologia 178(1):75-87

Folse HJ, Roughgarden J (2010) What is an individual organism? a multilevel selection perspective. Q Rev Biol 85(4):447-472

Forrest P (2016) The Identity of Indiscernibles. In: Edward N. Zalta (ed) The Stanford Encyclopedia of Philosophy (Winter 2016 Edition). https://plato.stanford.edu/archives/win2016/entries/identity-indis cernible/.

Freund J, Brandmaier AM, Lewejohann L, Kirste I, Kritzler M, Krüger A, Sachser N, Lindenberger U, Kempermann G (2013) Emergence of individuality in genetically identical mice. Science 340(6133):756-759

Gärtner KA (2012) Third component causing random variability beside environment and genotype. A reason for the limited success of a 30 year long effort to standardize laboratory animals? Int J Epidemiol 41:335-341

Gayon J (1998) The concept of individuality in Canguilhem's philosophy of biology. J Hist Biol 31(3):305-325

Godfrey-Smith P (2009) Darwinian populations and natural selection. OUP, Oxford

Godfrey-Smith P (2015) Individuality and Life Cycles. In: Guay A, Pradeu T (eds) Individuals across the sciences. OUP, Oxford, pp 85-103

Gorelick R (2012) Mitosis circumscribes individuals; sex creates new individuals. Biol Philos 27(6):871-890

Gould SJ, Lloyd EA (1999) Individuality and adaptation across levels of selection: how shall we name and generalize the unit of Darwinism? PNAS 96:11904-11909

Griesemer J (2018) Individuation of developing systems: a reproducer perspective. In: Bueno O, Chen R-L, Fagan MB (eds) Individuation, process, and scientific practices. OUP, New York, pp 137-164

Guay A, Pradeu T (2015) To be continued: the genidentity of physical and biological processes. In: Guay A, Pradeu T (eds) Individuals Across the Sciences. OUP, Oxford, pp 317-347

Haber M (2015) The biological and the mereological. In: Guay A, Pradeu T (eds) Individuals Across the Sciences. OUP, Oxford, pp 295-316

Hauskeller C (2004) Genes, genomes and identity. Projections on matter. New Genet Society 23(3):285-299

Hawley K (2009) Identity and indiscernibility. Mind 118(469):101-119

Herron MD, Rashidi A, Shelton DE, Driscoll WW (2013) Cellular differentiation and individuality in the 'minor' multicellular taxa: differentiation and individuality. Biol Rev 88(4):844-861

Hull DL (1978) A Matter of Individuality Philos Sci 45(3):335-360

Huneman P (2014) Individuality as a Theoretical Scheme. I. Formal and Material Concepts of Individuality. Biol Theory 9(4):361-373

Kaiser MI (2018) Individuating Part-Whole Relations in the Biological World. In: Bueno O, Chen R-L, Fagan MB (eds) Individuation, Process and Scientific Practices. OUP, Oxford, pp 63-87

Kaiser MI (2019) Normativity in the philosophy of science. Metaphilosophy 50(1-2):36-62

Kaiser MI, Müller C (2021) What is an animal personality? Biol Philos. https://doi.org/10.1007/s1053 9-020-09776-w

Kaiser M I, Trappes R (forthc.) Individual-level Mechanisms in Ecology and Evolution. In: Love A C, Baxter J, Bausman W, Lean O, and Waters C K (eds) From Biological Practice to Scientific Metaphysics, vol. 23. University of Minnesota Press. Minneapolis.

Kovaka K (2015) Biological individuality and scientific practice. Philos Sci 82(5):1092-1103

Krause D, Arenhart JRB (2016) Individuality, quantum physics, and a metaphysics of non-individuals. The role of the formal. In: Guay A, Pradeu T (eds) Individuals Across the Sciences. OUP, Oxford, pp 61-84

Layman CA, Newsome SD, Crawford TG (2015) Individual-level niche specialization within populations: emerging areas of study. Oecologia 178:1-4

Lidgard S, Nyhart LK (2017) The Work of Biological Individuality: Concepts and Contexts. In: Lidgard S, Nyhart LK (eds) Biological Individuality Integrating. Scientific, Philosophical and Historical Perspectives. University of Chicago Press, Chicago, pp 17-62

Love A C (2005) Explaining Evolutionary Innovation and Novelty: A Historical and Philosophical Study of Biological Concepts. Dissertation, University of Pittsburgh.

Love AC (2008) Explaining evolutionary innovation and novelty: criteria of adequacy and multidisciplinary prerequisites. Philos Sci 75:874-886 
Love AC (2016) Explaining the origins of multicellularity: between evolutionary dynamics and developmental mechanisms. In: Niklas KJ, Newman SA (eds) Multicellularity: Origins and Evolution. MIT Press, Cambridge, MA, pp 277-296

Love AC, Brigandt I (2017) Philosophical dimensions of individuality. In: Lidgard S, Nyhart LK (eds) Biological Individuality. Integrating, Scientific Philosophical and Historical Perspectives. University of Chicago Press, Chicago, pp 318-348

Lowe EJ (2003) Individuation. In: Loux MJ, Zimmerman DW (eds) The Oxford Handbook of Metaphysics. OUP, Oxford, pp 75-95

Lowe EJ (2016) Non-individuals. In: Guay A, Pradeu T (eds) Individuals Across the Sciences. OUP, Oxford, pp 49-60

Maienschein J (2011) 'Organization' as setting boundaries of individual development. Biol Theory 6(1):73-79

Maynard Smith J, Szathmary E (1995) The major transitions in evolution. OUP, Oxford

McConwell A (2017) Contingency and individuality: a plurality of evolutionary individuality types. Philos Sci 84:1104-1116

Minelli A (2011) Animal development, an open-ended segment of life. Biol Theory 6(1):4-15

Minelli A (2020) Biological individuality: a complex pattern of distributed uniqueness. In: Pennisi A, Falzone A (eds) The Extended Theory of Cognitive Creativity: Interdisciplinary Approaches to Performativity. Springer, Cham, pp 185-198

Novak M, Tinker MT (2015) Timescales alter the inferred strength and temporal consistency of intraspecific diet specialization. Oecologia 178:61-74

Nyhart LK, Lidgard S (2017) Introduction: working together on individuality. In: Lidgard S, Nyhart LK (eds) Biological Individuality, Integrating Scientific, Philosophical and Historical Perspectives. University of Chicago Press, Chicago, pp 1-16

Oliveira RF, Taborsky M, Brockmann HJ (2008) The evolution of alternative reproductive tactics: concepts and questions. In: Oliveira RF, Taborsky M, Brockmann HJ (eds) Alternative reproductive tactics: an integrative approach. Cambridge University Press, Cambridge, pp 1-21

Pâslaru V (2018) Mechanisms in ecology. In: Glennan S, Illari P (eds) The Routledge Handbook of Mechanisms and Mechanical Philosophy. Routledge, New York, pp 348-361

Pepper JW, Herron MD (2008) Does biology need an organism concept? Biol Rev 83(4):621-627

Pradeu T (2012) Limits of the Self. OUP, New York

Pradeu T (2016) The many faces of biological individuality. Biol Philos 31(6):761-773

Pradeu T (2018) Genidentity and biological processes. In: Nicholson DJ, Dupré J (eds) Everything Flows: Towards a Processual Philosophy of Biology. OUP, Oxford, pp 96-112

Sachser N, Hennessy M B, Kaiser S (2018) The adaptive shaping of social behavioral phenotypes during adolescence. Biol Lett, 14(11).

Santelices B (1999) How Many Kinds of Individual Are There? Trends Ecol Evol 14(4):152-155

Sih A, Bell A, Johnson JC (2004) Behavioral syndromes: an ecological and evolutionary overview. Trends Ecol Evol 19(7):372-378

Simondon G (1992) The genesis of the individual. Cohen M, Kwinter S (trans). Incorporations 6:297-319

Smith-Ferguson J, Beekman M (2019) Can't see the colony for the bees: behavioural perspectives of biological individuality. Biol Rev 94:1935-1946

Strawson PF (1959) Individuals: An Essay in Descriptive Metaphysics. Routledge, London and New York Trappes, R (2021) Individuality in Behavioural Ecology. https://doi.org/10.17605/OSF.IO/RKU47

Tremmel M, Müller C (2013) Insect personality depends on environmental conditions. Behav Ecol 24(2):386-392

Trillmich F, Guenther A, Müller C, Reinhold K, Sachser N (2015) New perspectives in behavioural development: Adaptive shaping of behaviour over a lifetime? Frontiers in Zoology 12(1):S1

Waters CK (2018) Ask Not 'What Is an Individual?' In: Bueno O, Chen R-L, Fagan MB (eds) Individuation, Process, and Scientific Practices. OUP, New York, pp 91-113

Wilson J (1999) Biological Individuality: The Identity and Persistence of Living Entities. CUP, New York

Wilson R A, Barker M (2018) The Biological Notion of Individual. In: Zalta E N (ed) The Stanford Encyclopedia of Philosophy (Summer 2018 Edition). https://plato.stanford.edu/archives/sum2018/ entries/biology-individual/

Wolf M, Weissing FJ (2012) Animal personalities: consequences for ecology and evolution. Trends Ecol Evol 27:452-546

Wong AH, Gottesman I, Petronis A (2005) A. Phenotypic differences in genetically identical organisms: the epigenetic perspective. Hum Mol Genet 14(1):R11-R18 
Violle C, Enquist BJ, McGill BJ, Jiang L, Albert CH, Hulshof CM, Jung V, Messier J (2012) The return of the variance: intraspecific variability in community ecology. Trends Ecol Evol 27(4):244-252

Vogt G, Huber M, Thiemann M, van den Boogaart G, Schmitz OJ, Schubart CD (2008) Production of different phenotypes from the same genotype in the same environment by developmental variation. J Exp Biol 211:510

Vogt G (2015) Stochastic developmental variation, an epigenetic source of phenotypic diversity with farreaching biological consequences. J Biosci 40:159-204

Publisher's Note Springer Nature remains neutral with regard to jurisdictional claims in published maps and institutional affiliations. 